\title{
SIMULATION METHOD TO ESTIMATE THE UNCERTAINTIES OF ISO SPECIFICATIONS
}

\author{
J.M. LINARES , J.M. SPRAUEL
}

Aix Marseille Université, CNRS, ISM UMR 7287, 13288 Marseille, cedex 09, France

In this work a simplified method, dedicated to the use in industrial environments, is proposed to evaluate uncertainties of ISO 1101 specifications. For that purpose a Delete d-Jack-knife Method is implemented and adapted to the estimation of specification uncertainties. As example, the verification uncertainty of a ISO geometrical specification will be presented. The advantages and limitations of the method are then discussed.

\section{Introduction:}

ISO/IEC 17000 Standard [1] defines accreditation as an "Attestation issued by a third party related to a conformity assessment body conveying formal recognition of its competence to carry out specific conformity assessment tasks". ISO/IEC 1702 specifies and defines the general terms relating to conformity assessment, including the accreditation of conformity assessment bodies, and the use of conformity assessment to facilitate trade. Recently, the accreditation for 3D measures of ISO specifications was launched in European countries [2]. This accreditation imposes to estimate the uncertainties of ISO 1101 geometrical specifications [3], and this even in case of measurements carried out in industrial environments. In this work a simplified method, dedicated to the use in industrial environments, is therefore proposed to evaluate uncertainties of ISO 1101 geometrical specifications $[4,5]$. For that purpose a Delete d-Jack-knife method is implemented and adapted to the estimation of specification uncertainties. As example, the verification uncertainty of ISO specification will be presented. The advantages and limitations of the method will then be discussed.

\section{Uncertainty propagation methods:}

\subsection{GUM:}

Uncertainties are generally evaluated using classical GUM's method [6] which is based on specific laws of propagation. A first order Taylor series expansion is also used to propagate elementary uncertainties to the composed 
uncertainty of the measurand. This propagation method has nevertheless some limitations, principally, when the model of the measurand is non linear. In such case, indeed, the shape of the PDF is distorted and some bias is observed for the calculated mean value of the result.

\subsection{Monte Carlo Simulation Method:}

Recently, a supplement to the GUM (GUM S1) has shown how to overcome this problem by using the Monte Carlo simulation Method (MCM) to evaluate uncertainties. MCM is a computational algorithm that relies on repeated random sampling to obtain numerical results and derive statistical parameters (mean value, standard deviation) [7-8]. MCM is a common tool in uncertainty evaluation of complex measurement processes. It is used because of the lack or the difficulty to express analytical solutions. The convergence rate of Monte Carlo methods is $O(1 / \sqrt{N})$, where $N$ is the number of simulated experiments. Instead of using pseudo-random generators, it can be accelerated by employing deterministic uniformly distributed sequences known as presenting lowdiscrepancy. Methods based on such sequences are named Quasi Monte Carlo. Asymptotically, Quasi Monte Carlo can provide a rate of convergence of about $O(I / N)[9]$. MCM needs however numerous repeated random sampling and thus often leads to large Tables.

\subsection{Sobol's method:}

In analytical propagation approach, the sensitivity coefficient may also be defined by Sobol's approach [10]. This method [11] is a variance based global sensitivity analysis technique founded upon "Total Sensitivity Indices" that account for interaction effects of the variables. The Total Sensitivity Indices of an input is defined as the sum of all the sensitivity indices involving that input. This method includes both main effect of each input as well as the interactions with the other variables [12]. Sobol's method can cope with both nonlinear and non-monotonic models, and provides a truly quantitative ranking of inputs and not just a relative qualitative measure. Effort has been done to reduce the computational complexity associated with the calculation of Sobol's indices. However, even with its most recent developments, Sobol's method remains computer time consuming.

\subsection{Jack-knife, Bootstrap or delete d-Jack-knife methods:}

To reduce, the computing time of MCM, the Jack-knife, Bootstrap or delete d-Jack-knife methods can be used to estimate the uncertainties of ISO standard specifications [13, 14]. 
The jack-knife was thought up by Quenouille in 1949. Ten years after, Tukey has developed its use in statistics. This method requires less computational power than MCM. For a dataset $x=\left(x_{1}, x_{2}, \ldots, x_{n}\right)$ of size $n$ and an estimator $\hat{\theta}$, the Jack-knife derives estimators $\hat{\theta}_{i}$ on subsamples that leave out a given selected element $x_{i}$. The subsample is defined by this equation.

$$
x_{(i)}=\left(x_{1}, x_{2}, \cdots, x_{i-1}, x_{i+1}, \cdots, x_{n}\right)
$$

The size of each Jack-knife subsample $x_{(i)}$ is $p=n-1$ and the total number of datasets that can be built is $n$. No sampling method is needed to define the subsamples. To estimate an uncertainty, the standard error of the Jack-knife replications is needed. Its estimate is defined by [15]:

$\hat{s}_{e}=\sqrt{\frac{n-1}{n} \sum_{i=1}^{n}\left(\hat{\theta}_{(i)}-\hat{\theta}_{(\bullet)}\right)^{2}}$ with $\hat{\theta}_{(\bullet)}=\frac{1}{n} \sum_{i=1}^{n} \hat{\theta}_{(i)}$

Generally, Jack-knife's method gives fine results for smooth statistics and for sufficiently large $\mathrm{n}$. Nevertheless, it does not give accurate estimations for nonsmooth statistic or nonlinear behavior.

The Bootstrap method was thought up after Jack-knife's method. B. Efron introduced it in 1979. For a dataset $x=\left(x_{1}, x_{2}, \ldots, x_{n}\right)$ of size $n$ and an estimator $\hat{\theta}$, the Bootstrap derives the estimator $\hat{\theta}$ on a resample $b$ of the same size $n$. Each resample is obtained by random sampling with replacement from the original dataset. The total number of resamples that can thus be built is $n^{n}$. A lower number $B$ of datasets is however used in practice to estimate uncertainties. It is usually fixed to $B=200$ for standard error estimation and $B=500$ for error bar estimation. The standard error of the estimator $\hat{\theta}$ can be derived from the Bootstrap replications using the equation $[13,15]$ :

$\hat{s}_{e}=\sqrt{\frac{1}{B-1} \sum_{b=1}^{B}\left(\hat{\theta}_{(b)}-\hat{\theta}_{(\bullet)}\right)^{2}}$ with $\hat{\theta}_{(\bullet)}=\frac{1}{B} \sum_{b=1}^{B} \hat{\theta}_{(b)}$

The delete d-Jack-knife method consists in generating subsamples, simply by randomly removing a number $d$ of elements from the initial dataset. The size of each subsample is thus $n-d$. The total number of subsamples that can be built is the number of combinations of $d$ elements removed from the original dataset of size $n$. As compared to the earlier Jack-knife scheme the delete d-Jack-knife sub-sampling technique leads thus to a greater number of sub-datasets. This can improve the accuracy of the method in the case of non-smooth statistics [16]. The standard error of the estimator $\hat{\theta}$ can be evaluated through this equation [15]: 


$$
\hat{s}_{e}=\sqrt{\frac{n-d}{d .\left(\begin{array}{l}
n \\
d
\end{array}\right)} \sum_{i=1}^{n}\left(\hat{\theta}_{(i)}-\hat{\theta}_{(\bullet)}\right)^{2}} \text { with } \hat{\theta}_{(\bullet)}=\frac{1}{\left(\begin{array}{l}
n \\
d
\end{array}\right)} \sum_{i=1}^{\left(\begin{array}{l}
n \\
d
\end{array}\right)} \hat{\theta}_{(i)}
$$

To obtain an accurate estimate of the standard error, the number $d$ of deleted data elements has to be selected in the range: $\sqrt{n} \leq d \leq n-1$.

An overview of other potential methods that can be used to estimate the uncertainties of geometrical specification has been presented in this section. A modified delete d-jack-knife method was finally chosen in our study to evaluate the measurement uncertainty of an ISO 1101 specification. The results of this work will be developed in the next section.

\section{Estimation of the verification uncertainty of a geometrical specification using a modified Jack-knife method:}

\subsection{Geometrical specification checking:}

Figure 1 shows an example of parallelism constraint as specified with ISO standard. The tolerance zone which defines the limits of the checked surface is bounded by two planes parallel to the datum plane A. In the example of figure 1 which deals with the parallelism between the specified surface and the datum plane A, the geometrical defect to be evaluated and checked is defined by the distance between the two planes that bound the measured points $M_{j}$, while being parallel to the datum feature A characterized by the digitized coordinates $M_{i}$. In a given reference frame $(O, X, Y, Z)$ these requirements are expressed by the minimisation conditions:

$$
\left\{\begin{array}{l}
\min \left[\max \left(e_{i}\right)-\min \left(e_{i}\right)\right] \\
\min \left[\max \left(e_{j}\right)-\min \left(e_{j}\right)\right] \\
\text { while } \vec{n}=\overrightarrow{n_{A}} \\
\text { where }: \\
e_{i}=\overrightarrow{M_{i}} \cdot \overrightarrow{n_{A}} \\
e_{j}=\overrightarrow{O M_{j}} \cdot \vec{n}
\end{array}\right.
$$

This equation needs two optimisation steps. The initial optimisation that corresponds to the first minimization condition permits to determine the normal 
vector $\vec{n}_{A}$ of the datum plane A. The final step which is expressed by the second minimization condition defines the parallelism defect between the specified surface and the datum feature A. The cosines of the normal vectors of the specified surface and the datum surface are imposed equal. In consequence and after best fit, the value of minimax criterion of distances between the measured points and the specified surface describes the value of parallelism defect. These calculations allow thus to evaluate the parallelism defect of the checked surface. Next section will now focus on the presentation of the method used to estimate the uncertainty of this value.

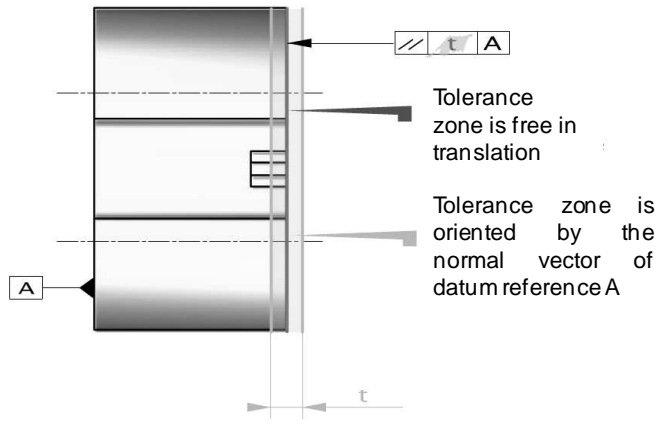

Figure 1. ISO Parallelism Specification.

\subsection{Modified Jack-knife method:}

The orientation of the datum surface A is, greatly, influenced by the outlier measured points. This fact can lead to a non smooth statistic of the searched parallelism defect. As stated in section 2.4, the Delete d-Jack-knife permits to accurately estimate standard errors even in the case of non-smooth statistics [16]. This method was therefore chosen to estimate the verification uncertainty of the parallelism defect.

In the classical Delete d-Jack-knife method, the number of deleted points is usually fixed to a given value $d$ that remains the same for all subsamples. In the proposed method, on the contrary, this number $d$ was selected randomly. In order to contain sufficient statistical information, both the datum plane and the specified surface were characterized by datasets of at least 25 acquisition points. For each sub-sampling, the modified Delete d-Jack-knife consisted then in a random generation of two subsets of the initial data: one constructed with the coordinates that define the datum plane and one created with the points acquired to characterize the specified feature. 


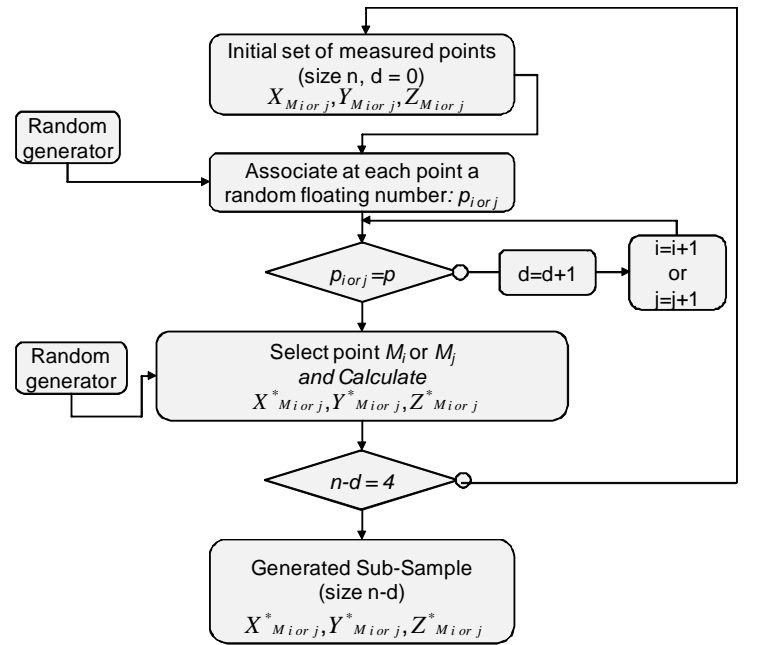

Figure 3. Sample and uploading acquisition uncertainty

The smallest number of points $M_{i}$ or $M_{j}$ required to build these two subsamples was fixed to 4 that is one more than the minimal number needed to define a plane ( 3 points). A specific selection procedure was implemented to generate the two sub-datasets. For every sub-sampling sequence, it consisted in randomly associating a floating number to each element of the initial datasets, that was built in the range $[0,1]$. A cut-off threshold $p$ was then fixed to only select the points with a linked value lower than this limit. A random perturbation was finally added to each coordinate to account for the calibration uncertainty of the Coordinate Measuring Machine (CMM). ISO 10360 standard [17] was used for this last operation. This standard permits to know the calibration error bar $\Delta$ for a measured length $L$ in the CMM volume. For a CMM, this value can be defined by this equation:

$$
\Delta= \pm(a+b \cdot L)
$$

The measured length $L$ was derived from the coordinates of the acquired points $M_{i}$ or $\mathrm{M}_{j}$. This equation was then applied to add random perturbations to the three initial coordinates which permits to account for the acquisition error $\Delta$ of the CMM. These calculations assume a uniform probability density of the calibration errors in the range $[-\Delta, \Delta]$ : 


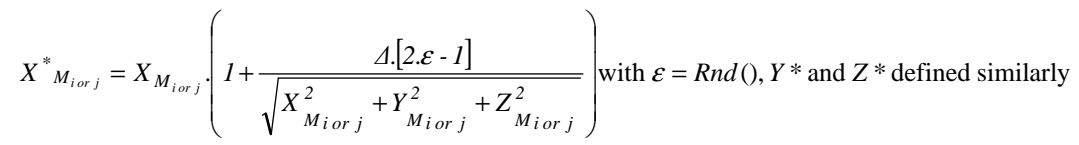

In these relationships, $R n d()$ is the random generation of a uniformly distributed variable in the interval $[0,1]$. The whole procedure used to build each sub-sample is presented in figure 3. After optimisation based on different tests, the value of the cut-off threshold $p$ was chosen to 0.4 . This value guarantees to obtain sub-samples of sufficient number of points.

\subsection{Error Bar of ISO specification:}

The uncertainties of a given specification are defined by the standard error of the geometrical defect to be characterized. It is based on repeated random generation of subsamples of the datum and specified surfaces using the modified d-Jack-knife procedure. After estimation of the mean value of the geometrical defect to be checked, the initial set of points is replicated by the random d-Jackknife method already presented. At each replication step, the geometrical defect of the virtual surfaces associated to the generated datasets is then computed. This operation is repeated $\mathrm{M}$ times. The standard error of the set of values which are thus obtained if finally calculated. It represents the uncertainty of the estimated geometrical defect. The lower bound of the tolerance interval of orientation specifications is always equal to 0 . A test was therefore implemented in the calculation of the error bars of the estimated geometrical defect to avoid negative values of the confidence interval starting point. If this check detects a negative indicator, a unilateral distribution of probability is considered to define the error bars. The lower bound of the confidence interval is then fixed to 0 . A bilateral probability distribution is considered otherwise.

\section{Conclusion:}

Uncertainty calculation of ISO specifications is a complex task. In industrial context, the calculation time is a main constraint. The revised version of the GUM (GUM S1) proposes to use Monte Carlo simulations for the evaluation of uncertainties, but this method is much computer time consuming. To avoid this impediment, an alternative uncertainty calculation method was proposed. It is based on a modified delete d-jack-knife sub-sampling technique. The results obtained with this method for measured surfaces with a small form defect are in complete adequacy with ISO 1101 standard for parallelism specification. The orientation of datum surface was deduced tangent of the material using the minimax criterion and the error bar of ISO specification was deduced quickly using the modified Delete-d-jack-knife method. 


\section{References}

1. ISO/IEC 17000:2004, Conformity assessment - Vocabulary and general principles.

2. http://www.mesures.com/pdf/old/816-Dossier-Metrologie-3.pdf

3. ISO 1101: Third edition 2012, Geometrical product specifications (GPS) Geometrical tolerancing -Tolerances of form, orientation, location and runout.

4. Ricci, F., Scott, P.J., Jiang X., 2013, A categorical model for uncertainty and cost management within the Geometrical Product Specification (GPS) framework Precision Engineering 37, p.265- 274.

5. Maihle, J., Linares, J.M., Sprauel, J.M., 2009, The statistical gauge in geometrical verification: Part I. Field of probability of the presence of matter, Precision Engineering 33, p.333-341.

6. BIPM, IEC, ISO, IUPAC, IUPAP, OIML; "Guide to the expression of the uncertainty in measurement, First Edition". 1993, ISBN 92-6710188-9.

7. Wen, X.L., Zhao, Y.B., Pan, J., 2013, Adaptative Monte Carlo and GUM methods for evaluation of measurement uncertainty of cylindricity error. Precision Engineering 37, p.856- 864.

8. Linares, J.M., Sprauel, J.M., Bourdet, P., 2009, Uncertainty of reference frames characterized by real time optical measurements: Application to Computer Assisted Orthopaedic Surgery. CIRP Annals - Manufacturing Technology 58, p.447-4.50

9. Søren Asmussen and Peter W. Glynn, Stochastic Simulation: Algorithms and Analysis, Springer, 2007, 476 pages.

10. Allard, A. and Fischer N., 2009, Sensitivity analysis in metrology: study and comparison on different indices for measurement uncertainty, Advanced Mathematical and Computational Tools in Metrology and Testing VIII, World Scientific, p1-6.

11. Sobol, I.M., 1993, Sensitivity estimates for nonlinear mathematical models, Mathematical Modelling and Computation 1, p.407-414.

12. Saltelli, A., 2002. Making best use of model evaluations to compute sensitivity indices, Computer Physics Communication 145, p.280-297.

13. Farooqui, S.A., Doiron, T., Sahay, C., 2009, Uncertainty analysis of cylindricity measurements using bootstrap method, Measurement 42, p.524531.

14. Efron, B., 1993. An Introduction to the Bootstrap. Chapman \& Hall.

15. Rapacchi, B., Une introduction au bootstrap, Centre Interuniversitaire de Calcul de Grenoble, 1994, 74 pages.

16. Shao, J., Wu, C.F.J., 1989, A general-theory for jackknife varianceestimation, Annals of statistics 17/3, p.1176-1197.

17. ISO 10360 Part 2: 2005, Performance assessment of coordinate measuring machines. 